\title{
Making use of Point Cloud for Generating Subtractive Solar Envelopes
}

\author{
Miktha Farid Alkadri ${ }^{1}$, Francesco De Luca ${ }^{2}$, Michela Turrin ${ }^{3}$, \\ Sevil Sariyildiz ${ }^{4}$ \\ ${ }^{1,3,4}$ Chair of Design Informatics, Faculty of Architecture and the Built Environ- \\ ment, TU Delft ${ }^{2}$ Department of Civil Engineering and Architecture, Tallinn Uni- \\ versity of Technology \\ ${ }^{1,3,4}\{$ M.F.Alkadri|M.Turrin|I.S.Sariyildiz\}@tudelft.nl²francesco.deluca@ttu.ee
}

\begin{abstract}
As a contextual and passive design strategy, solar envelopes play a great role in determining building mass based on desirable sun access during the predefined period. With the rapid evolution of digital tools, the design method of solar envelopes varies in different computational platforms. However, current approaches still lack in covering the detailed complex geometry and relevant information of the surrounding context. This, consequently, affects missing information during contextual analysis and simulation of solar envelopes. This study proposes a subtractive method of solar envelopes by considering the geometrical attribute contained in the point cloud of TLS (terrestrial laser scanner) dataset. Integration of point cloud into the workflow of solar envelopes not only increases the robustness of final geometry of existing solar envelopes but also enhances awareness of architects during contextual analysis due to consideration of surface properties of the existing environment.
\end{abstract}

Keywords: point cloud data, solar envelopes, subtractive method, solar access

\section{INTRODUCTION}

Awareness to consider solar access during the development of built environment principally is taken into account since the Ancient period. This can be observed through several examples such as The Hanging Gardens during the Babylonian period in 605562 BCE (Cartwright 2018), El-Lahun village in Egypt (1857-1700 BC) with a checkerboard urban grid and narrow streets facing North-South (Mazzone 2017), and Classical Greek cities in 4th Century BC utilizing the idea of solar oriented homes (Butti and Perlin 1980). These principles ultimately drive further
\end{abstract}

the concept of solar envelopes proposed by Knowles (1974). As a contextual and passive design strategy, solar envelopes consist of imaginary building mass determined by considering the amount of desirable sun access without violating surrounding buildings during the predefined period. With a principle of space-time constraint (Knowles 1981), input parameter of solar envelopes can be divided into geographic properties (plot, the distance of surrounding buildings, and shadow fences) and climatic properties (site location such as latitude and longitude, and time limit). These parameters play an important role in de- 
termining the volumetric size of solar envelopes.

Furthermore, the evolution of digital tools affects design platforms and methods of solar envelopes, ranging from conventional techniques such as descriptive, profile angle, and 2D orthographic projection (Topaloglu 2003) to computational methods including descriptive geometry (DG), solar obstruction angle (SOA), constructive solid geometry (CSG), and digital elevation modelling (DEM) (Alkadri et al 2018). However, these current methods pose several barriers that may result in missing information during contextual analysis. In most cases, for example, the existing 3D site modelling (e.g., solid modelling (Staneva 2008)) lacks in preserving complex geometry of surrounding context especially when it comes to the isolated and dense areas. Consequently, relevant site properties such as vegetation, material and other temporal site elements are often neglected during the simulation of solar envelopes.

One of the fascinating methods is subtractive form finding mechanism (De Luca, 2017) in which refers to CSG. This method generates solar envelopes by using volumetric samples of three-dimensional matrix or the so-called 3D polyhedral. In principle, the polyhedra are extruded from the proposed land parcel based on criteria of new building such as width and height, functional utilities, number of floors and the like. Ideally, every 3D polyhedron can be associated with one typical room in the real building. This subtractive method, furthermore, involves sun visibility to identify visible sun hours during a selected period. In this way, each polyhedron acts as a 3D voxel carrying information relative to sun access of neighbouring buildings. However, the mechanism of sun visibility becomes challenging when dealing with blocked areas from the sun. The current approach of context modelling merely focuses on the self-blocked mechanism from the existing building's geometry so as to predominantly neglect site properties that might be relevant for sunlight hours simulation (e.g., vegetation). Besides, the existing approach also uses centroid points that represent surrounding windows during ray tracing analysis. These window samples are, however, limited to calculate the entire area of surrounding facades. This study, therefore, attempts to improve the existing workflow of solar envelopes by making use of potential application of point cloud data in capturing the real context.

As a product of 3D laser scanning, point cloud has been extensively used regarding data representation and data analysis in various disciplines such as civil engineering, computer science, photogrammetry, geoscience, and heritage. As a data structure, point cloud indicates a collection of multidimensional points (Randall, 2013). It is usually characterized by spatial XYZ coordinates and is optionally be assigned by typical attributes such as reflection intensity (I) (Weinmann, 2016), color properties (RGB) (Fujita, Hoshino, Ogata, \& Kobayashi, 2015), and any additional abstract information. These attributes are, furthermore, used to perform ray tracing analysis, substituting the role of centroid points on surrounding windows. Inclusion of surrounding properties during the simulation will expand the result analysis comprehensively.

In general, the proposed method in this study contributes to several aspects of the cycle of architectural design practices as follows:

- The well-informed site allows architects to construct more possibilities regarding simulation of solar envelopes such as solar collector and other microclimatic analysis. The proposed method simultaneously enhances awareness of architects during contextual analysis so that environmental properties of existing context can be paid more attention within the conceptual design phase.

- Integration between 3D scanning technology and solar envelopes may increase the robustness of the result analysis due to consideration of surface properties of the existing environment.

Furthermore, the following section will describe the computational procedure of the proposed method. 
Figure 1

Computational procedure of subtractive solar envelopes

Figure 2

The selected site and 3D polyhedra (land parcel)

\section{METHOD}

This study proposes a computational workflow for generating subtractive solar envelopes based on point cloud data (see Fig.1). In general, the computational procedure is categorized into four sections consisting of input, task, digital tools, and output. Description of each section is discussed in detail below.

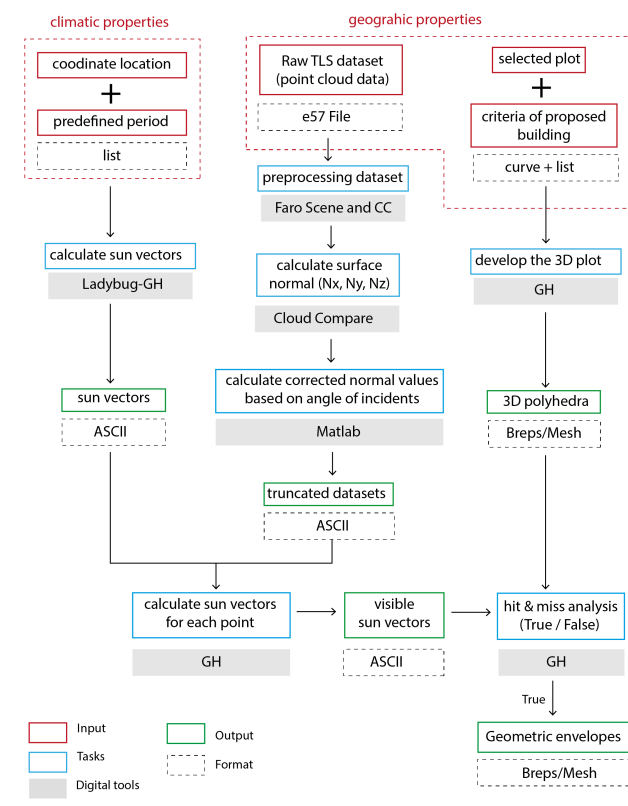

\section{Input}

In principle, all input is originated from climatic and geographic properties. Climatic properties are used to calculate the number of sun vectors that will be simulated on each point cloud during the simulation. It is produced according to a specific location within a certain period. In this case, the selected site is located in Groningen, Netherlands with latitude position $53.2194^{\circ} \mathrm{N}$ and longitude $6.5665^{\circ} \mathrm{E}$. The time setting takes a sample of the required period on $21 \mathrm{st}$ for each month from May to September, starting from 9 am to $9 \mathrm{pm}$. With the time step of 2, this setting results in around 25 sun vectors. Meanwhile, geometric properties involve two parts: the proposed building and surrounding environments. The proposed building is represented by 3D polyhedra that consist of 300 polyhedron (see Fig.2). Each polyhedron indicates the dimension of one typical room in the real building, consisting of $3 \times 3 \times 3 \mathrm{~m}$. It is placed in front of the existing building.

For the surrounding environment, this study employs a small sample of point cloud dataset. It consists of a small portion of building facades from TLS (terrestrial laser scanning) dataset of Middlestum Church in Groningen, Netherlands. The dataset is collected by using Faro Focus 3D laser scanner with wavelength $950 \mathrm{~nm}$. It is also supported by Nikon D5300 for capturing colour properties. The use of TLS datasets principally aims at obtaining more accurate representation, high-resolution formats, and broader coverage of isolated areas in comparing with ALS (airborne LiDAR) datasets. The data provided through these inputs are elaborated within the developed workflow.

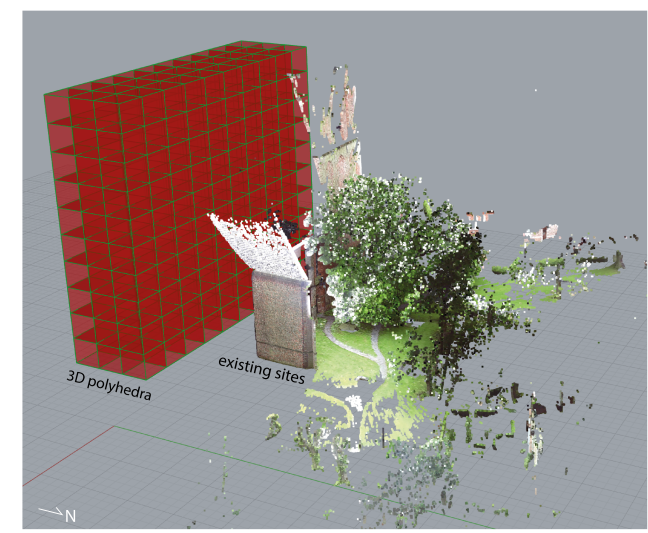

\section{Tasks}

This section contains a series of specific operations to generate solar envelopes. Some of these tasks can be performed simultaneously such as calculation of 
solar vectors and development of the 3D plot (polyhedra) due to simple construction procedures, while task of dataset pre-processing and normal values correction need to run sequentially due to the requirement of preliminary input. Some of these tasks are briefly discussed as follows:

- Dataset pre-processing.This task includes outlier (unnecessary cloud of points) removal, dataset subsampling, and conversion of the dataset's format. The use of dataset subsampling permits us to adjust the density of points so as to reduce time consumption during the simulation. In this case, the dataset is subsampled by $5 \mathrm{~cm}$ for the distance between points. Accordingly, it results in approximately 449.267 points from 31.5 million of points

- Calculation of surface normal. In order to calculate the normal direction of each point within the dataset, we apply Hough Normal plugin (Boulch \& Marlet, 2016) in Cloud Compare (CC) for an unstructured point cloud. In this case, several tolerance angles are simulated to set a variety of normal values from the angle of incidences, ranging from $10^{\circ}$ to $90^{\circ}$. A detailed procedure of this section has been addressed in our previous work (Alkadri, Turrin, \& Sariyildiz, 2019)

- Calculation of optimal normal values. In principle, some points within a certain angle can correspond very well to the projection of the laser beam during scanning. This affects the distribution of points that show point characteristics at a certain angle. Thus, an evaluation of the scattering points is performed by only keeping the densest cloud from all angles. It simultaneously aims at minimizing erroneous level regarding environmental factors during scanning. In this case, the dataset truncation is set to range between $0-0.01$ that results in proximately 239.178 total points to be selected for optimal normal values (see Fig.3).



Figure 3

The truncation of the dataset
- Calculation of sun visibility. This step aims at calculating visible sun vectors that will be applied to each point within the dataset. It is done by multiplying sun vectors from the indicated period with optimal normal vectors from truncated points. In order to obtain points that meet the criteria of sun visibility, these vectors are subsequently filtered by considering only values that are smaller than the projected angle of $90^{\circ}$. This is because values that are equal and larger than $90^{\circ}$ consist of zero and negative cosine values, respectively. It means that those values exclude within the list of visible sun vectors.

- Ray tracing analysis. This part consists of ray tracing procedure or hit \& miss analysis between selected sun vectors, points and, the 3D polyhedra (300 polyhedron). Figure 4A illustrates that selected points for ray tracing analysis are primarily originated from the building façade due to optimized normal values of the dataset. This calculation results in around 110, 7 million of intersections (see Fig. 4B). With a Boolean operation, voxels with "True" values will be used to generate solar envelopes while "False" values are indicated as an obstruction that needs to be eliminated (see Fig. 4C). 
Figure 4

Ray tracing procedure A - Hit \& miss analysis $B$ -

Points obstruction $C$ - Voxels intersection
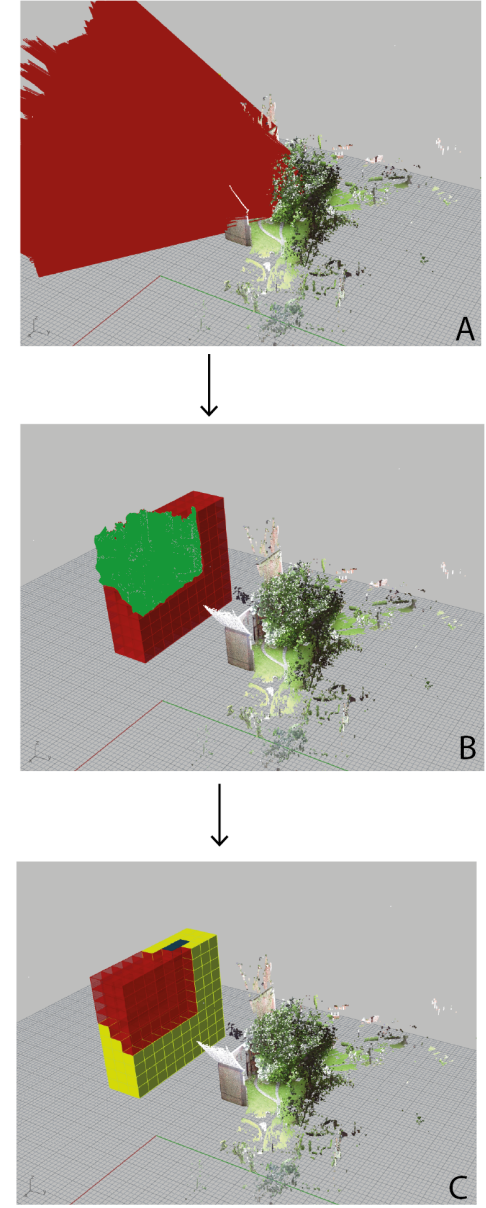

\section{Digital tools}

In general, this study employs various digital tools depending on specific tasks performed within the computational workflow. For examples, 3D modelling and environmental simulation during the generation of solar envelopes are predominantly supported by Rhino and Ladybug component in Grasshopper. Meanwhile, dataset preparation and calculation of surface normal of point clouds in different angles are performed by Faro Scene and Cloud Compare (CC), respectively. Lastly, Matlab permits us to calculate optimal normal values and make a truncation for selected datasets.

\section{Output}

According to the workflow presented in Fig.1, several outputs have been generated during the process of simulation depending on the performed specific task in the workflow. These outputs then become input for the following procedure such as sun vectors, 3D polyhedra, truncated datasets, and visible sun vectors. The final output is geometric envelopes that successfully meet the criteria of solar envelopes.

\section{RESULT AND DISCUSSION}

As a result of the simulation (see Fig.5), this study presents several findings regarding the final geometry of solar envelopes as follows:

- The total voxels (polyhedron that carries information of sun vectors) that successfully meet the criteria for solar envelopes are 208 out of 300. It means that around 92 voxels are considered as obstruction geometries. These obstructive voxels are subsequently eliminated because of blocking direct sun access to surrounding facades during the indicated period. According to Figure 5, those voxels are averagely located on the above of the 4th floor but remains three full blocks on the Westside. This is because solar vectors produced by predefined cut-off-times and the site location are predominantly toward the Western direction with the incident angle approximately laying between $30^{\circ}-60^{\circ}$. In principle, this result can be more robust if we densify solar vectors during the simulation. For example, by increasing the number of cut-off times allows us not only to increase the number of solar vectors but also to generate a broader incident angle so as to widen intersection coverage. This, however, will require high computational cost and 


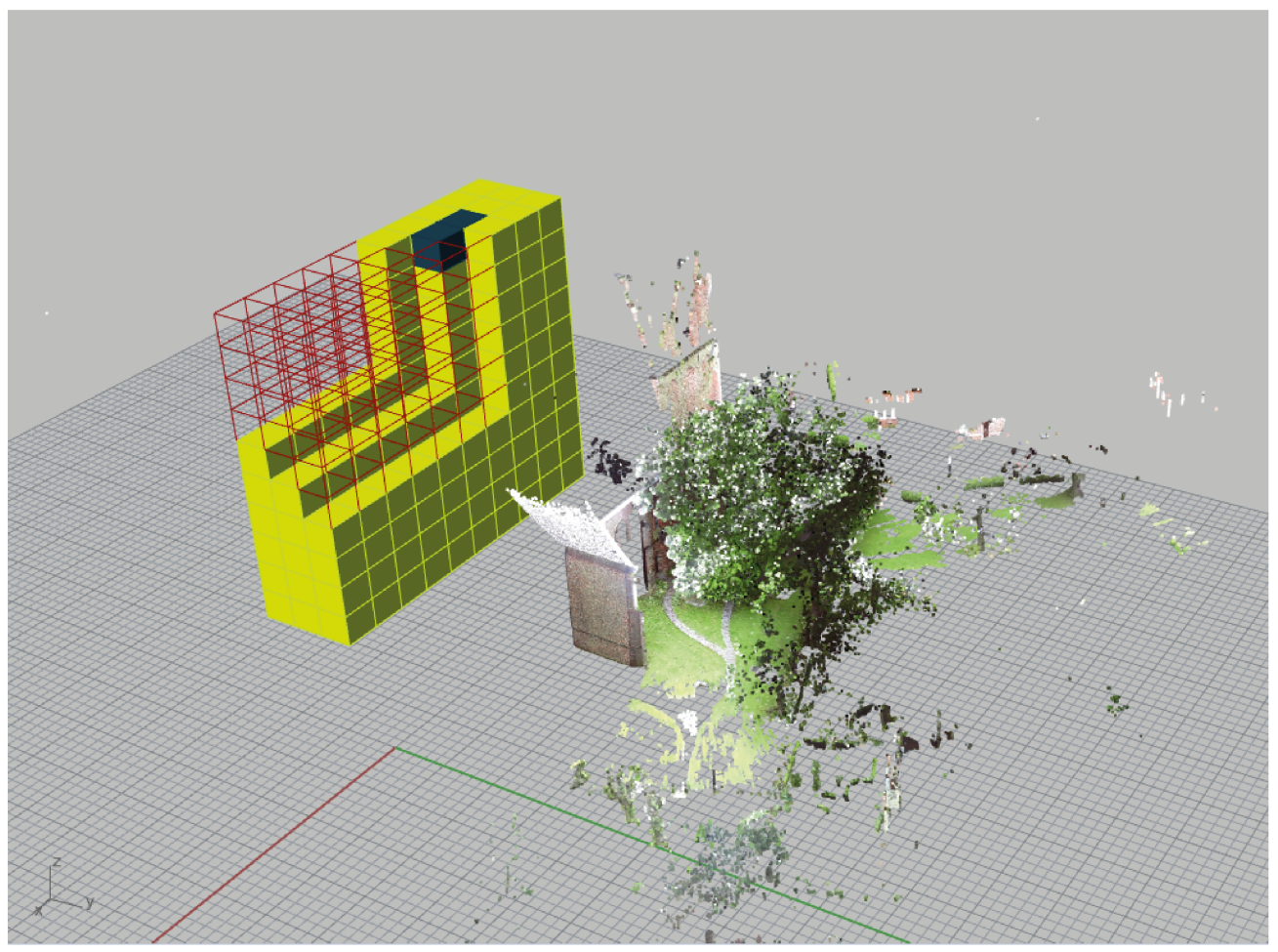

Figure 5

The final geometry of solar envelopes 
time due to hundreds of millions of rays intersection.

- The remaining voxels constitute colour-coded values that show the level of obstruction index, ranging from yellow with 0 obstructions to the light green with medium obstruction and the last, dark green with a high level of obstruction. In this case, Figure 5 illustrates that the polyhedra predominantly consists of yellow colour. Only two voxels are being identified containing medium obstruction index, which is located on the top of the polyhedra. It means that these voxels are potentially violating direct sun access to the surrounding building within this period. When it comes to functional utilities, these remaining voxels can be filled with any building's program that needs direct sun access while for the removed one and light green voxels may be filled with open space and courtyard, respectively.

\section{CONCLUSION AND FUTURE RECOMMEN- DATION}

This study proposes a novel method of subtractive solar envelopes by making use of $3 \mathrm{D}$ point cloud data. The proposed method aims at not only compensating missing information during contextual analysis but also integrating existing computational workflow of solar envelopes with geometrical properties of 3D scanning technology. According to the proposed workflow, several concluding remarks can be drawn as follows:

- The use of point cloud data in solar envelopes allows us to extend the functional properties of 3D scanning technology into the architectural design stage especially related to environmental analysis rather than merely as data visualization.

- Calculation of optimal normal values during the dataset processing is used not only to obtain corrected values of point cloud but also to minimize the erroneous level of datasets dur- ing scanning.

- Subtractive mechanism of point cloud confirms the feasibility of the proposed method to deal with solar access by including site properties of the existing environment.

- The proposed method can be a starting point to raise awareness of public policies especially related to contextual analysis and microclimate condition for the future development of sustainable architecture and the built environment.

However, some acknowledge limitations need further consideration. For example, the computational issue during the simulation remains a great barrier to perform a huge amount of the dataset. Alternatively, some aspects need particular adjustments such as the number of sun vectors and dataset subsampling. Besides, the inclusion of complex urban properties may enhance the analysis result of simulation such as street, urban scale, and the distance between the land parcel and existing building. This is important because these aspects may influence the intersection of total rays during the simulation so that it can affect the final geometry of solar envelopes.

Furthermore, this study highlights some potential aspects for future recommendation. For example, radiometric information contained in point cloud data can be further explored to integrate material properties of the existing environment with the proposed solar envelopes. This can simultaneously complement environmental performance simulation of new solar envelopes regarding the contextual analysis of the built environment. Moreover, the proposed method can be further explored by using a different climatic context such as tropical countries. This can expand a wide range of design implementation of solar envelopes with different urban settings.

\section{ACKNOWLEDGEMENT}

The research described in this paper was part of Ph.D research which financially supported by Indonesian Endowment Fund for Education (LPDP). The authors would like to thank to Valentini Vanhecke (4Visualiza- 
tion) for providing access to the dataset.

\section{REFERENCES}

Alkadri, MF, Turrin, M and Sariyildiz, S 2018, 'The use and potential application of point clouds in simulation of solar radiation for solar access in urban contexts', Advances in Computational Design, 3, pp. 319-338

Alkadri, MF, Turrin, M and Sariyildiz, S 2019, 'A computational workflow to analyse material properties and solar radiation of existing contexts from attribute information of point cloud data', Building and Environment, 155, pp. 268-282

Boulch, A and Marlet, R 2016 'Deep Learning for Robust Normal Estimation in Unstructured Point Clouds', Proceeding of SGP 2016, Berlin, pp. 281-290

Butti, K and Perlin, J 1980, A Golden Threat 2500 Years of Solar Architecture and Technology, Van Nostrand Reinhold Company

Fujita, Y, Hoshino, Y, Ogata, S and Kobayashi, I 2015, 'Attribute assignment to point cloud data and its usage', Global journal of computer science and technology, 15, pp. 11-18

Knowles, RL 1974, Energy and form: An ecological approach to urban growth, The MIT Press

Knowles, RL 1981, Sun, rhytm and form, The MIT Press

De Luca, F 2017 'Solar form finding. Subtractive Solar Envelope and Integrated Solar Collection Computational Method for High-rise Buildings in Urban Environments', Proceeding of ACADIA 2017 Conference DISCIPLINES+DISRUPTION, Cambridge (MA), pp. 212-221

Mazzone, D 2017, 'The Dark Side of a Model Community: The 'Ghetto' of el-Lahun', The Journal of Ancient Egyptian Architecture, 2, pp. 19-54

Randall, T 2013, Client guide to 3D scanning and data capture, BIM Task Group

Staneva, NN 2008, 'Approaches for generating 3D solid models in AutoCAD and solid works', Journal of Engineering, $\mathrm{Vl}$, pp. 28-31

Topaloglu, B 2003, Solar envelope and form generation in architecture, Graduate School of natural and applied science of the middle east of technical university

Weinmann, M 2016, Reconstruction and analysis of 3D scenes, Springer International Publishing

[1] https://www.ancient.eu/Hanging_Gardens_of_Baby

lon/ 